\title{
Theoretical and experimental investigations of polyelectrolyte adsorption dependence on molecular weight
}

Fei Xie*, Hongduo Lu*, Tommy Nylander**, Lars Wågberg*** and Jan Forsman*

*Theoretical Chemistry, Lund University, P.O.Box 124, S-221 00 Lund, Sweden

**Physical Chemistry, Lund University, P.O.Box 124, S-22100 Lund, Sweden ***Wallenberg Wood Science Center, KTH Royal Institute of Technology, S-100 44

Stockholm, Sweden

E-mail: fei.xie@teokem.lu.se

Examples of how the ellipsometry measured adsorption of short chain PVNP on silica substrate varies with time are provided in SS1. The adsorption curves are reproducible, and the stable, clear plateau adsorption values were found in every salt concentrations.

${ }^{*}$ To whom correspondence should be addressed 

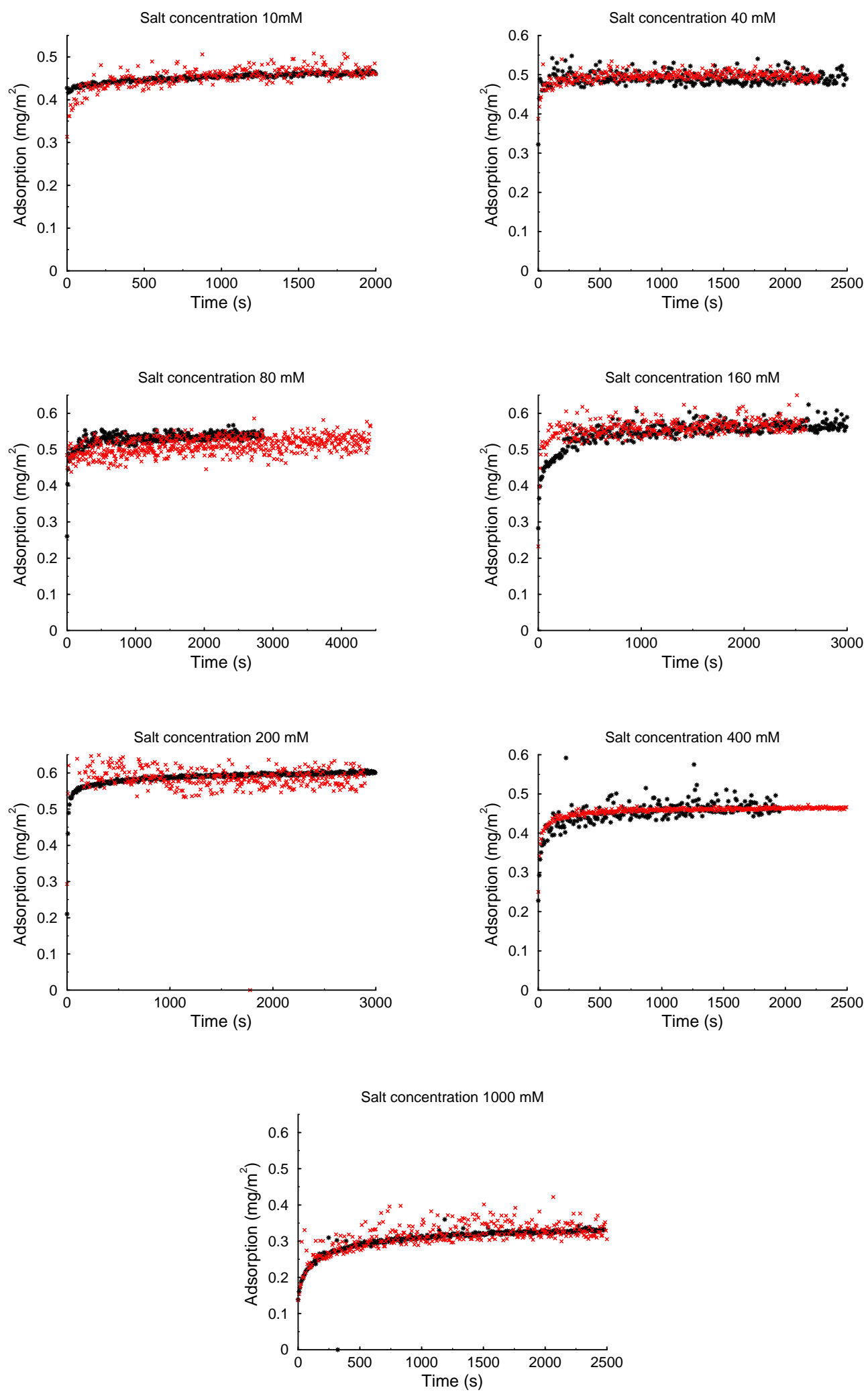

Figure S1: Adsorbed amount of short chain PVNP on silica surface as a function of time at different $\mathrm{NaCl}$ concentration at $\mathrm{pH}$ 9. Red and black curves indicate two reproducible measurements. 
Examples of how the ellipsometry measured adsorption of long chain PVNP on cellulose surfaces varies with time are provided in SS2. 

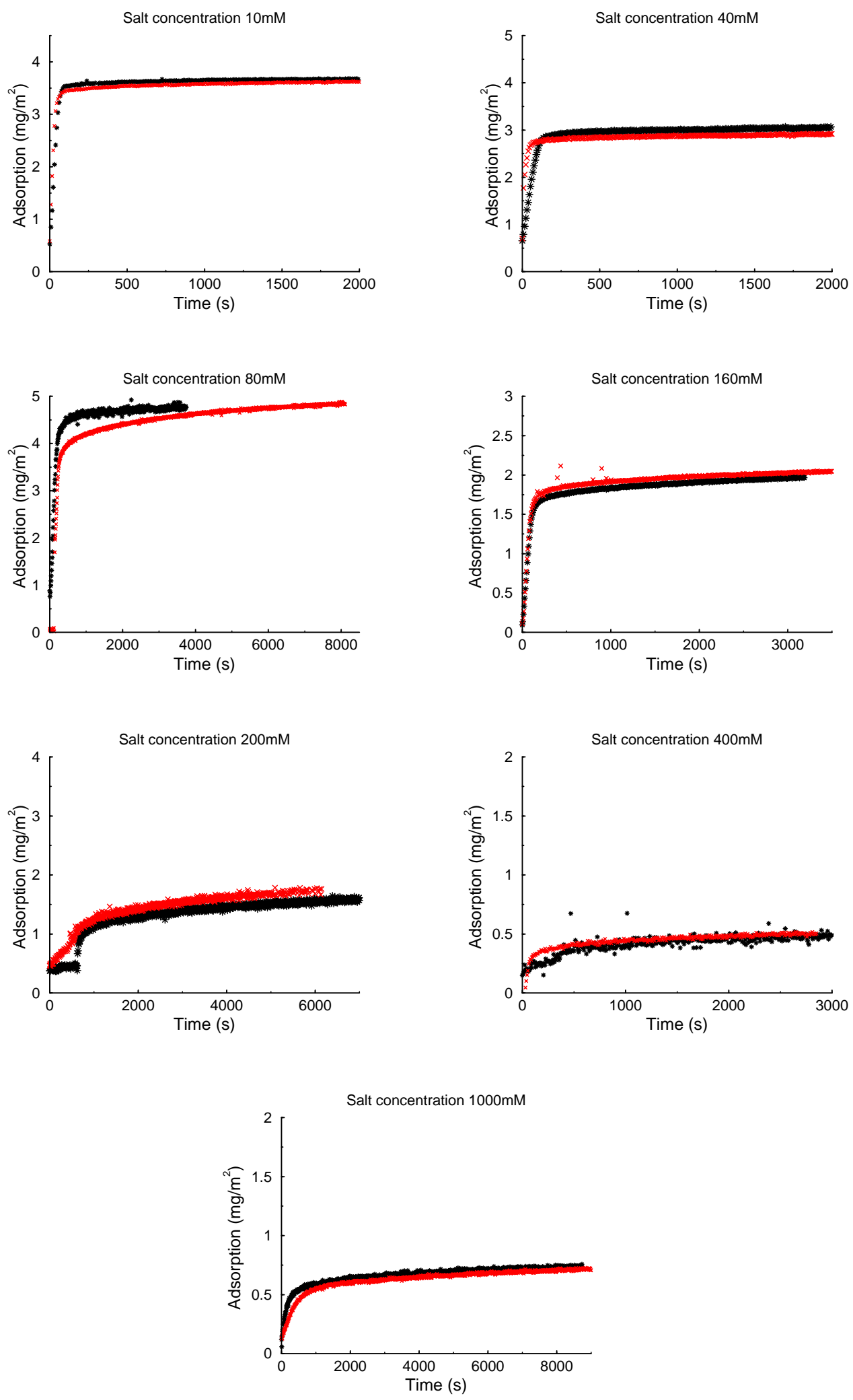

Figure S2: Adsorbed amount of long chain PVNP on cellulose surface as a function of time at different $\mathrm{NaCl}$ concentration at $\mathrm{pH}$ 9. Red and black curves indicate two reproducible measurements. 
Examples of how the ellipsometry measured adsorption of short chain PVNP on cellulose surfaces varies with time are provided in SS3. In the higher salt concentrations, e.g. 200mM, $400 \mathrm{mM}$ and $1000 \mathrm{mM}$, we adsorption curves look reproducible and reach to the plateau values. However, in the low salt regime, from $10 \mathrm{mM}$ to $160 \mathrm{mM}$, it is hard to get the equilibrium adsorption data, the adsorption describe a monotonic increase in some salt concentration (40mM, 80mM and 160mM). This may indicate that the short chain PVNP has tendency to penetrate into the cellulose thus make the equilibrium process really slow. 

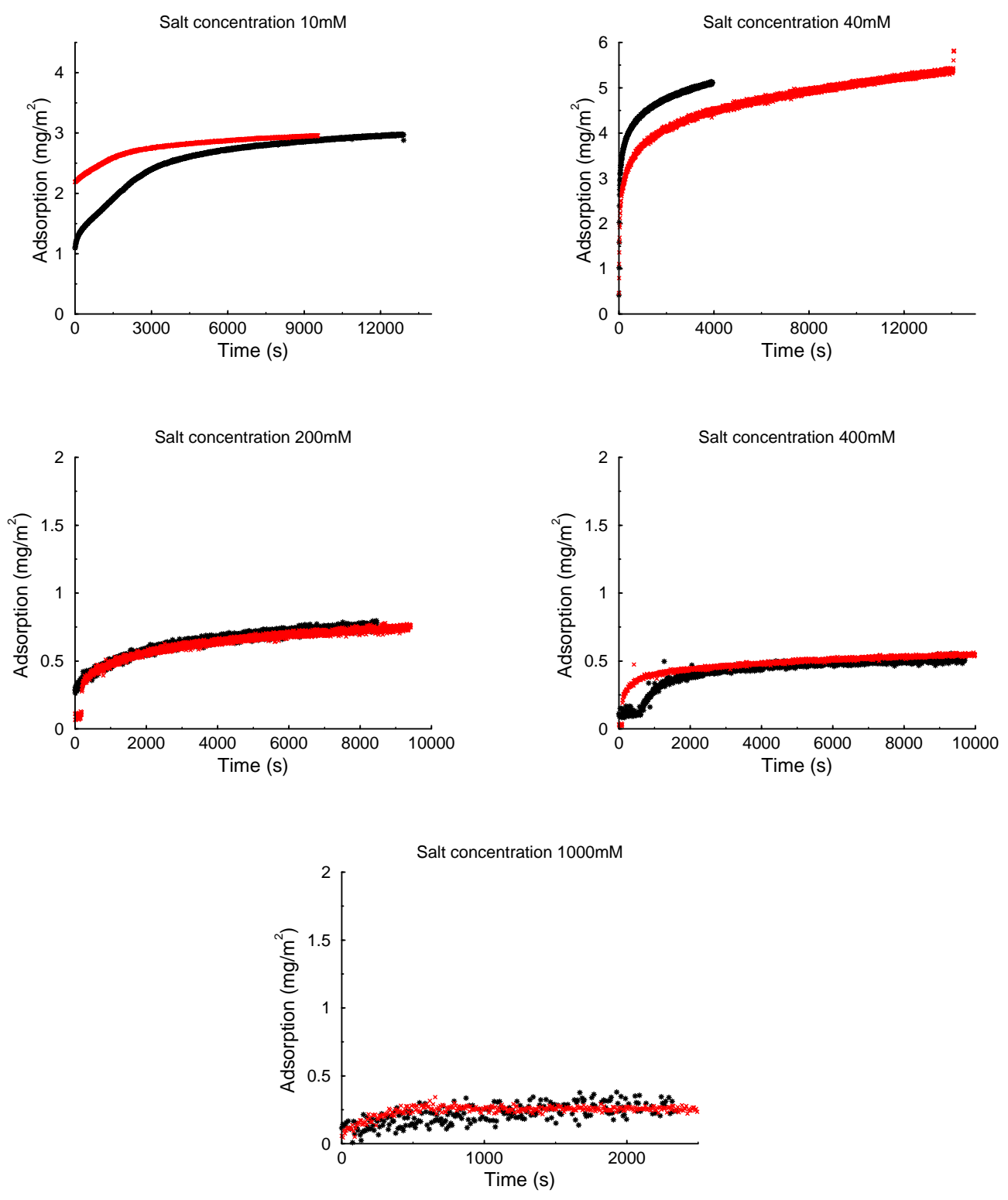

Figure S3: Adsorbed amount of short chain PVNP on cellulose surface as a function of time at different $\mathrm{NaCl}$ concentration at $\mathrm{pH}$ 9. Red and black curves indicate two reproducible measurements. 